\title{
MOBILITY-ASSISTED COOPERATIVE LOCALIZATION SCHEME FOR WIRELESS SENSOR NETWORKS
}

\author{
Hongyang Chen ${ }^{1}$, Pei Huang ${ }^{2}$, Hing Cheung So ${ }^{3}$, Xi Luo ${ }^{4}$, and Ping Deng ${ }^{4}$ \\ ${ }^{1}$ Institute of Industrial Science, The University of Tokyo, Tokyo, Japan \\ ${ }^{2}$ Department of Computer Science and Engineering, Michigan State University, USA \\ ${ }^{3}$ Department of Electronic Engineering, City University of Hong Kong, Hong Kong, China \\ ${ }^{4}$ Institute of Mobile Communications, Southwest Jiaotong University, China
}

\begin{abstract}
In this paper, we propose a novel cooperative localization algorithm which can be effectively used in mobility-assisted wireless sensor networks (WSNs). We propose an attractive movement strategy with mobile elements (MEs) to improve the network performance and address limitations of static WSNs in node localization. In our scheme, a mobile sensor node cooperates with static sensor nodes and moves actively to construct virtual anchor nodes. The localization accuracy of the proposed algorithm is further improved by active movement of ME with low communication traffic and computing complexity. It minimizes the communication cost and improves the localization performance simultaneously via using ME and our proposed virtual anchor nodes. Simulation results demonstrate the efficiency of our proposed algorithm.
\end{abstract}

Index Terms-Mobility-assisted wireless sensor networks, Mobile elements, Centroid localization algorithm, Cooperative localization scheme.

\section{INTRODUCTION}

Localization algorithms for wireless sensor networks (WSNs) have been designed to find per-node location information, which is a key technology to satisfy the application requirements of WSNs. Generally speaking, based on the type of information required for the positioning, protocols can be divided into two categories: (i) range-based and (ii) range-free protocols. Range-based protocols $[2,4,5]$ employ absolute point-to-point distance or angular information to identify locations of nodes. Some ranging techniques that are available for localization include angle-of-arrival (AOA) [6], received signal strength indicator (RSSI) [6], time-of-arrival (TOA) [2] and time-difference-of-arrival (TDOA) [5] schemes. On the other hand, we may adopt range-free approaches in which one can find the locations of unknown-position nodes by using the connectivity information with the designated but sparse anchor nodes with known positions, also known as landmarks. Due to the hardware limitations and power constraints of sensor nodes, solutions of range-free localization are often more preferable and can be considered as cost-effective options when compared with those more expensive and energy-consuming range-based schemes [3]. In this paper, we will focus on the investigation of range-free localization algorithms for mobility-assisted WSNs.

In order to increase the localization performance without increasing much communication cost, we propose a novel cooperative localization scheme using the virtual anchor nodes and mobile element to refine the localization performance, which is different from the related former

978-1-4244-2677-5/08/\$25.00 C2008 IEEE works. Aiming to improve localization performance, not only the information between unknown-position nodes and anchor nodes but also the information between anchor nodes and MEs are efficiently used in our proposed scheme. Taking advantage of extra information between anchor nodes and MEs, the proposed cooperative localization scheme is expected to achieve high localization accuracy. In fact, simulation results demonstrate the superiority of the new design with ME.

This paper makes three major contributions to the localization problem in WSNs. First, we present a practical, fast and easy-to-use method to construct and calculate the position of virtual anchor node, which can be used to improve localization performance with ME. Using this method, not only the useful anchor nodes can be increased but also the distribution of anchor nodes can be optimized. Second, we propose a volume based centroid method which can effectively improve localization performance with relatively low communication traffic and computing complexity. Third, an attractive movement strategy for cooperative node is proposed to reduce the total moving distance of ME while satisfying the high localization performance, which can efficiently extend the ME lifetime.

The rest of the paper is organized as follows. Section II introduces the related work for this paper. Section III describes the details of the new cooperative localization scheme, including its derivation. In Section IV, theoretical performance for our method is deduced. In Section V, simulation results are reported and a comparative study of the localization performance is conducted. Finally, Section VI gives the concluding remarks.

\section{RELATED WORK}

In this section, we review research most relevant to our work and indicate the characteristic of our algorithm. There are many techniques and schemes in the literature proposed for localization in WSNs [18].

In some related literatures, after the first step of coarse localization, some unknown-position nodes with coarse positions are selected to act as new anchor nodes to refine the location results. However, it will also increase the total communication cost of system. Liu et al. [8] proposed to use virtual anchor nodes to improve localization accuracy based on DV-Hop algorithm. But in their method, only those real nodes that have obtained positions from coarse localization will be selected as virtual anchor nodes. Therefore, those virtual anchor nodes are actually real communication capable nodes.

Several studies exploited the effect of mobile nodes on node localization for WSNs. In these methods, a small 
number of mobile devices referred to as mobile elements (MEs) roam about sensing fields and assist to improve localization performance. Luo et al. [9] proposed a TDOA-based localization algorithm for movement-assisted sensor networks. A mobile beacon is used to measure the mobility-differentiated TOA in [9], which will increase mobile beacon's communication cost. Based on mobile beacon, a RF-based localization algorithm and RSSI-based localization algorithm were presented in [10] and [11], respectively. Both [10] and [11] depend on RSSI-based range measurement, which do not perform well in large sensor networks. A complex method was processed in a centralized way for mobile beacon to improve localization performance in [11]. However, it will significantly increase extra computational complexity and communication cost for mobile beacon. Xiao et al. [16] proposed a distributed algorithm to locating sensor nodes using a single moving beacon. Three beacon movement patterns and their effect on location performance have been studied in [16]. However, [16] only used one mobile beacon and did not consider cooperation between mobile beacon and static anchor node. However, the broadcasting interval for method in [16] needs to be small to achieve the high location performance, it will increase the broadcasting time and reduce the lifetime for ME.

Different from former work on localization algorithms for mobility-assisted WSNs, we study the efficient node cooperation and movement planning strategies that achieve high localization performance.

\section{ALGORITHM DEVELOPMENT}

In this section, we are going to derive a novel node cooperative localization method for mobility-assisted WSNs.

\section{A. Development of Novel Cooperative Scheme for Mobility-assisted WSNs}

This subsection describes our proposed cooperative positioning algorithm for mobility-assisted WSNs in detail. As we know, the localization performance of the traditional localization algorithm for WSNs increases as the number of anchor nodes increases. In some application scenarios, however, it is impractical to increase the anchor node ratio as the hardware cost increases. Therefore, in those sparse WSNs areas, where anchor nodes are limited, we propose to use mobile element to improve localization performance.

First, the network model which is adopted by the proposed cooperative localization algorithm will be described as follows:

\section{Network Framework}

As shown in Fig. 1, $M$ is the target unknown-position node, $A_{0}$ and $A_{1}$ are the most closest and the second closest anchor nodes to $M$ (determined by comparing RSSI measurements), respectively. ME will move through the common sensing area from static anchor node $A_{0}$ and $A_{1}$ and also will broadcast its position periodically. We name $A_{2}$ and $A_{4}$ as the sojourn points when mobile element roams around the common sensing area of static anchor nodes $A_{0}$ and $A_{1}$. Here, $A_{0}$ and $A_{1}$ act as the cooperative partners for mobile element. The distance between $A_{0}$ and $A_{2}$ is $d_{3}$. When we use the communication link from anchor node $A_{0}$ to anchor node $A_{1}$, we can extend line $A_{1} A_{0}$ to $A_{3}$, the distance between $A_{0}$ and $A_{3}$ is set to be $d_{5}$. Herein, $A_{3}$ can be selected to serve as a virtual anchor node for cooperative localization. When we obtain the position of $A_{3}$, it can be used for localization of unknown-position node through cooperation with mobile element $A_{2}$. Note that we assume mobile element knows its current physical position and can be acted as an anchor node. Let the distance between anchor node $A_{1}$ and anchor node $A_{0}$ be $d_{2}$, the distance between anchor node $A_{1}$ and mobile element $A_{2}$ be $d_{1}$ and the distance between mobile element $A_{2}$ and virtual anchor node $A_{3}$ be $d_{4}$.We define $\theta$ as the angle between line $A_{1} A_{0}$ and line $A_{1} A_{2}$, which can be obtained by using the cosine rule.

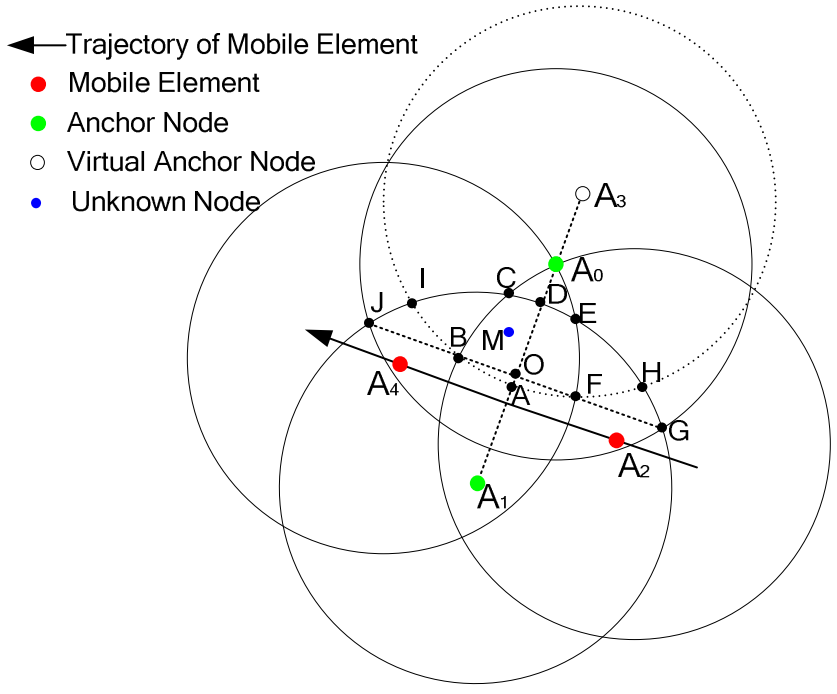

Fig.1. Network Model

Based on the above proposed scheme, one virtual anchor nodes are efficiently constructed. Furthermore, with modification of the distance of line $A_{0} A_{3}$, we can assure the unknown-position node $M$ is in the range of virtual anchor node $A_{3}$ (refer to Eq. (1)) when it is in some special areas. Then there are three extra anchor nodes, including virtual anchor node $A_{3}$, beacon points $A_{2}$ and $A_{4}$, which uniformly distributed around unknown-position node $M$ with its nearest anchor nodes $A_{0}$ and $A_{1}$ to further improve localization performance. It is a key advantage of our cooperative scheme. As we know, the more regularly anchors are placed, the lower the error and higher localization coverage for some state-of-art localization algorithms in WSNs.

The brief procedure of our cooperative scheme is introduced as follows: First, by comparing the received RSSI values from anchor nodes $A_{0}$ and $A_{1}$, 
unknown-position node $M$ realizes that anchor node $A_{0}$ is its closest anchor node. That means unknown-position node $M$ is constrained in the right side of line $B F$, which is the perpendicular for line $A_{1} A_{0}$ as shown in Fig.1. After obtaining positions of $A_{0}, A_{1}$ and $A_{2}$, the unknown-position node $M$ can calculate the values of $d_{3}, d_{2}$ and $d_{1}$. Then, unknown-position node $M$ can calculate the positions of virtual anchor nodes $A_{3}$. If $M$ can receive both beacon messages from $A_{2}$ and $A_{4}$, we consider the centroid of polygon $A B C D E F$ is the position of unknown-position node. The virtual anchor node will be utilized to estimate the node position under this situation since the unknown-position nodes is just in the range of virtual anchor node. As shown in Fig. $1, B, C, E, F, G, H, I$ and $J$ are intersections from the related pairs of circulars which are constructed based on anchor node, mobile element and virtual anchor node. $A$ and $D$ are intersections from line $A_{1} A_{0}$ to circulars based on centres of $A_{3}$ and $A_{1}$, respectively. However, if unknown-position node $M$ can only receive one beacon message from $A_{2}$ or $A_{4}$, we consider the centroid of polygon $E F G H$ or $B C I J$ is the estimated position of unknown-position node, respectively.

\section{Virtual anchor node positioning}

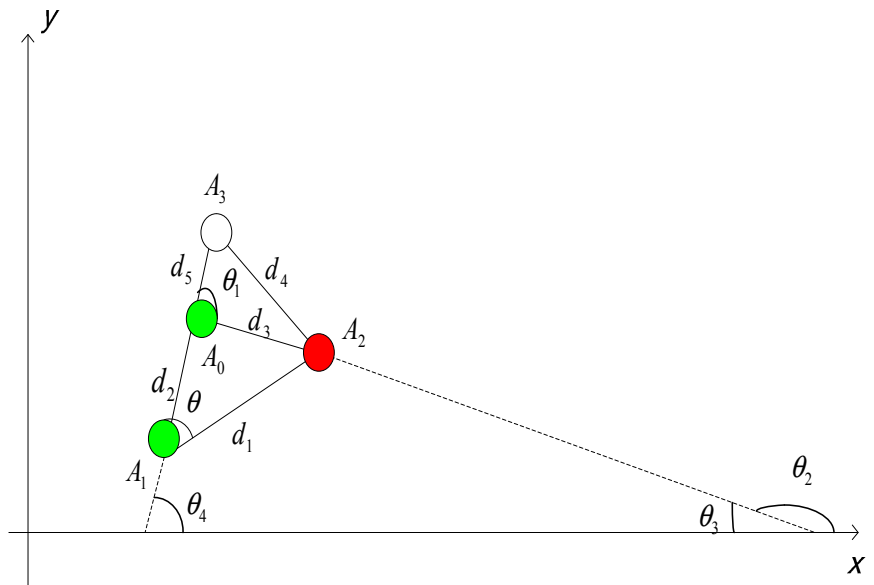

Fig.2. System Model

Let $\left(x_{0}, y_{0}\right),\left(x_{1}, y_{1}\right)$ and $\left(x_{2}, y_{2}\right)$ be the coordinates for anchor node $A_{0}$, anchor node $A_{1}$ and mobile element $A_{2} . O$ is the midpoint for line $A_{1} A_{0}$. In order to assure the unknown-position node $M$ is in the range of virtual anchor node $A_{3}$ when $M$ is in the area of polygon $A B C D E F$, we set the distance of $A_{0} A_{3}$ as :

$$
d_{5}=O A_{3}-O A_{0}=\sqrt{R^{2}-(O B)^{2}}-A_{0} A_{1} / 2
$$

Through the cooperation between anchor nodes $A_{0}, A_{1}$ and mobile element $A_{2}$, we obtain the values of $d_{2} d_{3}$ and $d_{1}$ based on the Euclidean distance formula:

$$
d_{1}=\sqrt{\left(x_{1}-x_{2}\right)^{2}+\left(y_{1}-y_{2}\right)^{2}}
$$

$$
\begin{aligned}
& d_{2}=\sqrt{\left(x_{1}-x_{0}\right)^{2}+\left(y_{1}-y_{0}\right)^{2}} \\
& d_{3}=\sqrt{\left(x_{2}-x_{0}\right)^{2}+\left(y_{2}-y_{0}\right)^{2}}
\end{aligned}
$$

After obtaining the values of $d_{2}, d_{3}$ and $d_{1}$ in triangle $\triangle A_{0} A_{1} A_{2}$, we get:

$$
\cos \theta=\frac{d_{2}^{2}+d_{1}^{2}-d_{3}^{2}}{2 d_{1} d_{2}} .
$$

Based on the values of $d_{2}, d_{1}, d_{3}$ and $\theta$ in triangle $\triangle A_{1} A_{2} A_{3}$, we obtain:

$$
\cos \theta=\frac{\left(d_{2}+d_{5}\right)^{2}+d_{1}^{2}-d_{4}^{2}}{2 d_{1}\left(d_{2}+d_{5}\right)}
$$

Since

$d_{2}+d_{1}+d_{5}>d_{4}, d_{2}+d_{5}+d_{4}>d_{1}, d_{1}+d_{4}>d_{2}+d_{5}$, we calculate the value of $d_{4}$ by (4) based on triangle $\Delta A_{1} A_{2} A_{3}$.

Then we get

$$
\theta_{1}=\arccos \left(\frac{d_{3}^{2}+d_{5}^{2}-d_{4}^{2}}{2 d_{3} d_{5}}\right)
$$

Furthermore, we have the slope of $A_{0} A_{2}, \mathrm{k}=\frac{y_{0}-y_{2}}{x_{0}-x_{2}}$. That is $\tan \theta_{2}=\frac{y_{0}-y_{2}}{x_{0}-x_{2}}$, where $\theta_{2}$ is defined as the angle of slope for line $A_{0} A_{2}$.

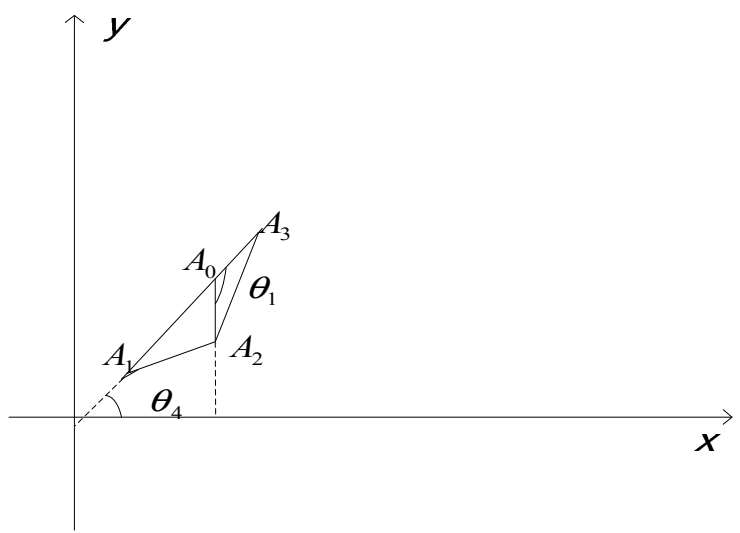

Fig.3. System Model

We consider various situations to obtain the value of $\theta_{4}$, which is defined as the angle of slope for line $A_{3} A_{1}$.

1. If $\theta_{2}=0^{\circ}$, we have $\theta_{4}=\theta_{1}$, which means line $M A_{1}$ is parallel to the axis $x$.

2. If $\theta_{2}=90^{\circ}$, we have $\theta_{4}=\theta_{1}+90^{\circ}$, where $0^{\circ}<\theta_{1} \leq 90^{\circ}$ (refer to Fig.4); If $90^{\circ}<\theta_{1}<180^{\circ}$, we get $\theta_{4}=\theta_{1}-90^{\circ}$ (refer to Fig.3).

3. If $0^{\circ}<\theta_{2}<90^{\circ}$ we 
have $\theta_{2}=\arctan \frac{y_{3}-y_{1}}{x_{3}-x_{1}}$ and $\theta_{4}=\theta_{1}+\theta_{2}$ (refer to Fig.5).

4. If

$$
90^{\circ}<\theta_{2}<180^{\circ}
$$

we

have

$$
\theta_{2}=\arctan \frac{y_{3}-y_{1}}{x_{3}-x_{1}}+180^{\circ}
$$

$\theta_{3}=180^{\circ}-\theta_{2}=-\arctan \frac{y_{3}-y_{1}}{x_{3}-x_{1}}$.Then we have

$\theta_{4}=\theta_{1}-\theta_{3}$ (refer to Fig.2).

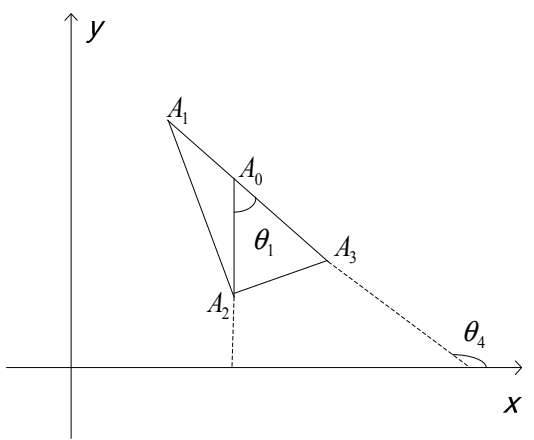

Fig.4. System Model

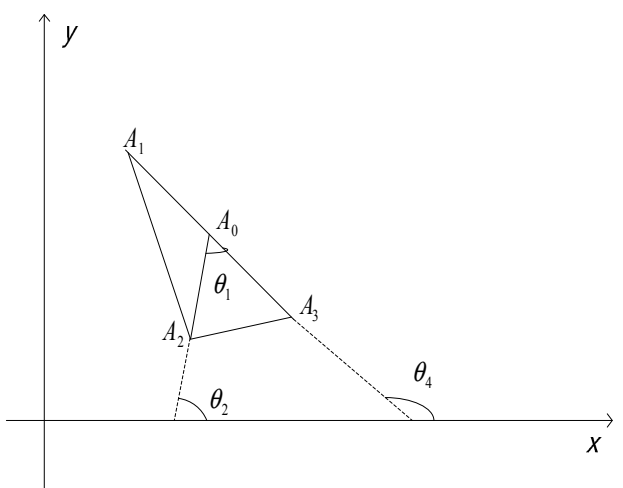

Fig.5. System Model

Using the values of $\theta_{4}, d_{3}$ and $d_{4}$, we get the equations related to position of $A_{3}(x, y)$ as follows:

$$
\left\{\begin{array}{c}
\left(x_{0}-x\right)^{2}+\left(y_{0}-y\right)^{2}=d_{5}^{2} \\
\left(x_{1}-x\right)^{2}+\left(y_{1}-y\right)^{2}=\left(d_{5}+d_{2}\right)^{2} \\
\left(x_{2}-x\right)^{2}+\left(y_{2}-y\right)^{2}=d_{4}^{2} \\
\frac{y-y_{1}}{x-x_{1}}=\tan \theta_{4}
\end{array}\right.
$$

(6)

$$
\text { Let } Z_{c}=\left[x, y, x^{2}+y^{2}\right]^{T} \text {, }
$$

$$
G_{c}=\left[\begin{array}{ccc}
-2 x_{0} & -2 y_{0} & 1 \\
-2 x_{1} & -2 y_{1} & 1 \\
-2 x_{2} & -2 y_{2} & 1 \\
\tan \theta_{4} & -1 & 0
\end{array}\right],
$$

and

$$
h_{c}=\left[\begin{array}{c}
d_{5}^{2}-x_{0}^{2}-y_{0}^{2} \\
\left(d_{5}+d_{3}\right)^{2}-x_{1}^{2}-y_{1}^{2} \\
d_{4}^{2}-x_{2}^{2}-y_{2}^{2} \\
x_{1} \tan \theta_{4}-y_{1}
\end{array}\right],
$$

by (8), we have

$$
G_{c} Z_{c}=h_{c}
$$

Using least squares (LS) algorithm [4], from (10) we get

$$
Z_{c}=\left(G_{c}^{T} G_{c}\right)^{-1} G_{c}^{T} h_{c}
$$

Then, the coordinates of the virtual anchor node $A_{3}$ $(x, y)$ is expressed as:

$$
\left\{\begin{array}{l}
x=Z_{c}(1), \\
y=Z_{c}(2) .
\end{array}\right.
$$

Based on the above procedure, we can effectively obtain the positions of virtual anchor nodes.

\section{Position Estimation}

In this subsection, we introduce the final position estimation method for unknown-position node. Our proposed localization algorithm works as follows:

1. Using our proposed cooperative localization scheme, we calculate the position of virtual anchor nodes via cooperation between target unknown-position node and neighbor cooperative partners.

2. The $i$ th unknown-position node, $i=1,2, \cdots, M$, calculate their own location by a centroid determination based on the positions of all real anchor nodes, mobile elements and virtual anchor nodes within their communication ranges.

Note that real anchor nodes, MEs and virtual anchor nodes will construct a complex area and it is difficulty to calculate the centroid of the polygon in this situation. To address this problem, we can divide this complex polygon into a series of tetrahedrons. Our idea is to calculate the centroid of these individual tetrahedrons first and then calculate the centroid of the polygon by combining these individual centroids using the center of mass.

Let $B_{k}\left(\hat{x}_{k}, \hat{y}_{k}, \hat{z}_{k}\right) \quad(k=1,2, \cdots, n)$ be the vertex of polygon $B_{1} B_{2} \cdots B_{k}$, we divide this polygon into $n-2$ tetrahedrons. Let the individual centroids of these tetrahedrons be $G_{k}\left(\bar{x}_{k}, \bar{y}_{k}, \bar{z}_{k}\right)$, the centroid of polygon $B_{1} B_{2} \cdots B_{k}, F(\bar{x} 2, \bar{y} 2, \bar{z} 2)$, is calculated as follows:

$$
\bar{x} 2=\frac{\sum_{i=1}^{n} m_{i} \bar{x}_{k}}{\sum_{i=1}^{n} m_{i}}, \bar{y} 2=\frac{\sum_{i=1}^{n} m_{i} \bar{y}_{k}}{\sum_{i=1}^{n} m_{i}}, \bar{z} 2=\frac{\sum_{i=1}^{n} m_{i} \bar{z}_{k}}{\sum_{i=1}^{n} m_{i}}
$$

For a continuous distribution with mass density $\rho$ and volume $v_{i}$ for the tetrahedron, we have:

$$
m_{i}=\rho v_{i}
$$

Substituting (14) into (13), we get: 


$$
\bar{x} 2=\frac{\sum_{i=1}^{n} v_{i} \bar{x}_{k}}{\sum_{i=1}^{n} v_{i}}, \bar{y} 2=\frac{\sum_{i=1}^{n} v_{i} \bar{y}_{k}}{\sum_{i=1}^{n} v_{i}}, \bar{z} 2=\frac{\sum_{i=1}^{n} v_{i} \bar{z}_{k}}{\sum_{i=1}^{n} v_{i}}
$$

The volume of the tetrahedron is given by:

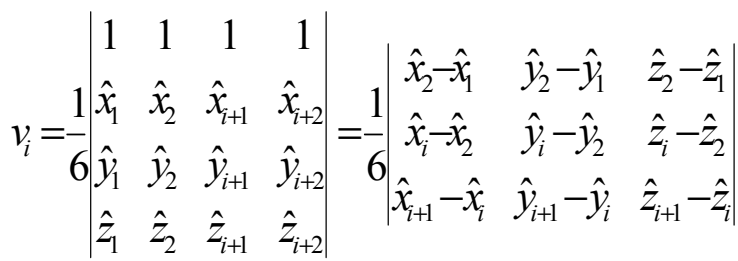

Thus, the centroid polygon $B_{1} B_{2} \cdots B_{k}, F(\bar{x} 2, \bar{y} 2, \bar{z} 2)$, can be calculated to estimate the position of node as shown in Eq. (17):

$$
\begin{gathered}
\bar{x} 2=\frac{\sum_{i=3}^{n-1}\left(\hat{x}_{1}+\hat{x}_{2}+\hat{x}_{i}+\hat{x}_{i+1}\right)\left|\begin{array}{ccc}
\hat{x}_{2}-\hat{x}_{1} & \hat{y}_{2}-\hat{y}_{1} & \hat{z}_{2}-\hat{z}_{1} \\
\hat{x}_{i}-\hat{x}_{2} & \hat{y}_{i}-\hat{y}_{2} & \hat{z}_{i}-\hat{z}_{2} \\
\hat{x}_{i+1}-\hat{x}_{i} & \hat{y}_{i+1}-\hat{y}_{i} & \hat{z}_{i+1}-\hat{z}_{i}
\end{array}\right|}{3 \sum_{i=3}^{n-1}\left|\begin{array}{ccc}
\hat{x}_{2}-\hat{x}_{1} & \hat{y}_{2}-\hat{y}_{1} & \hat{z}_{2}-\hat{z}_{1} \\
\hat{x}_{i}-\hat{x}_{2} & \hat{y}_{i}-\hat{y}_{2} & \hat{z}_{i}-\hat{z}_{2} \\
\hat{x}_{i+1}-\hat{x}_{i} & \hat{y}_{i+1}-\hat{y}_{i} & \hat{z}_{i+1}-\hat{z}_{i}
\end{array}\right|} \\
\bar{y} 2=\frac{\sum_{i=3}^{n-1}\left(\hat{y}_{1}+\hat{y}_{2}+\hat{y}_{i}+\hat{y}_{i+1}\right)\left|\begin{array}{ccc}
\hat{x}_{2}-\hat{x}_{1} & \hat{y}_{2}-\hat{y}_{1} & \hat{z}_{2}-\hat{z}_{1} \\
\hat{x}_{i}-\hat{x}_{2} & \hat{y}_{i}-\hat{y}_{2} & \hat{z}_{i}-\hat{z}_{2} \\
\hat{x}_{i+1}-\hat{x}_{i} & \hat{y}_{i+1}-\hat{y}_{i} & \hat{z}_{i+1}-\hat{z}_{i}
\end{array}\right|}{3 \sum_{i=3}^{n-1}\left|\begin{array}{ccc}
\hat{x}_{2}-\hat{x}_{1} & \hat{y}_{2}-\hat{y}_{1} & \hat{z}_{2}-\hat{z}_{1} \\
\hat{x}_{i}-\hat{x}_{2} & \hat{y}_{i}-\hat{y}_{2} & \hat{z}_{i}-\hat{z}_{2} \\
\hat{x}_{i+1}-\hat{x}_{i} & \hat{y}_{i+1}-\hat{y}_{i} & \hat{z}_{i+1}-\hat{z}_{i}
\end{array}\right|} \\
\hat{z} 2=\frac{\hat{x}_{i=3}^{n-1}\left(\hat{z}_{1}+\hat{z}_{2}+\hat{z}_{i}+\hat{z}_{i+1}\right)\left|\begin{array}{lll}
\hat{x}_{2}-\hat{x}_{1} & \hat{y}_{2}-\hat{y}_{1} & \hat{z}_{2}-\hat{z}_{1} \\
\hat{x}_{i}-\hat{x}_{2} & \hat{y}_{i}-\hat{y}_{2} & \hat{z}_{i}-\hat{z}_{2} \\
\hat{x}_{i+1}-\hat{x}_{i} & \hat{y}_{i+1}-\hat{y}_{i} & \hat{z}_{i+1}-\hat{z}_{i}
\end{array}\right|(17)}{3 \sum_{i=3}^{n-1}\left|\begin{array}{lll}
\hat{x}_{2}-\hat{x}_{1} & \hat{y}_{2}-\hat{y}_{1} & \hat{z}_{2}-\hat{z}_{1} \\
\hat{x}_{i}-\hat{x}_{2} & \hat{y}_{i}-\hat{y}_{2} & \hat{z}_{i}-\hat{z}_{2} \\
\hat{x}_{i+1}-\hat{x}_{i} & \hat{y}_{i+1}-\hat{y}_{i} & \hat{z}_{i+1}-\hat{z}_{i}
\end{array}\right|}
\end{gathered}
$$

Herein, we only focus our localization algorithm on twodimensional WSNs for simplicity. Thus, we delete the z-coordinate from the above equations. Finally, this calculated coordinates $(\bar{x} 2, \bar{y} 2)$ will be selected as the estimated position of unknown-position node in our localization algorithm.

\section{B. Movement Strategy for Mobile Element}

Due to the power constraint, a ME is only capable of low-speed and short-distance movement in real deployments For instance, the normal speed of several mobile sensor platforms (e.g., Packbot and XYZ) is only $0.5-2 \mathrm{~m} / \mathrm{s}$. A XYZ mobile sensor node can only move about 165 meters before exhausting its power, which is supported by two AA batteries [7]. Therefore, the movement trace of a ME must be efficiently planned in order to maximize the amount of target positions that can be obtained with high localization accuracy within a short moving distance. Moreover, scheduling an optimal path for the ME improves the system reliability and network lifetime.

To address the constraints mentioned above, we propose an effective moving strategy for MEs. We let MEs prefer moving to the areas which have lower density of anchor nodes. This balances the density of anchor nodes and makes the distribution of anchor nodes more uniform. At each sojourn point, a ME broadcasts its current position to stimulate nodes within its radio range to perform calculation of virtual anchor nodes. Anchor nodes overhearing the message reply to it with their own location information. With the anchor nodes' position information, the ME makes the next moving decision by establishing a coordinate system that takes its current position as the origin and divides the coordinate system into eight equal sectors. Assuming the ME knows the network periphery and the direction from which it moves to current position, it can mark the sectors that are out of the network region and the sector where it comes as invalid ones. This prevents the ME from moving out of the network region or moving back to the previous location.

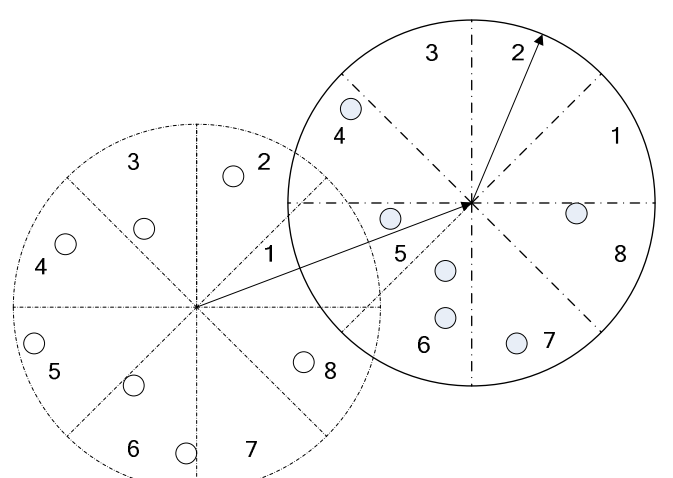

Fig.6. Movement strategy

Among the other valid sectors, the ME chooses the sector which has the smallest number of anchor nodes as the forward sector. As shown in Fig. 6, 1) if there exists a sector that has the smallest number of anchor nodes, it moves along the midline of the sector to the next sojourn point which is $4 r / 3$ away from the current position, where $r$ is the nominal radio range of the ME. 2) If several sectors have the same smallest number of anchor nodes, the ME combines those sectors that can be combined and moves along the midline of the combined sector (as illustrated in the right coordinate system of Fig. 6). 3) If several sectors have the same smallest number of anchor nodes and they cannot be combined, the ME chooses the sector whose left and right sectors cover the fewest anchor nodes as the forward sector (as shown in the left coordinate system of Fig. 6). 4) However, if both the left and right sectors also cover the same number of anchor nodes (e.g., an anchor node resides in sector 2 of the right coordinate system), the ME moves along the midline which has the largest angle with the direction from which it arrives at current position.

Based on this effective moving strategy, MEs pass through the areas which have relatively lower density of anchor nodes and thus help those nodes to perform the localization algorithm. In general, unnecessary movements of MEs are avoided. It can effectively decrease the total moving distance and number of broadcast to extend MEs' lifetime and improve the system reliability. performance analysis 


\section{Lower Bounds}

Nagpal et al. [12] proposed a theoretical bound for the accuracy of any range-free localization algorithm in statistic wireless sensor networks. Although M. Rudafshani et al. [13] pointed out some problems related to this lower bound. We can still utilize it to analyze the localization accuracy for our algorithm.

Mendenhall et al. [14] mentioned the probability that there are $k$ sensor nodes in a given area $\alpha$ satisfies a Poisson distribution:

$$
P=\frac{(\rho \alpha)^{k}}{k !} e^{-\rho \alpha}
$$

where $\rho$ is equal to $n / s, n$ is the total number of sensor nodes and $s$ is the related surface area. The main idea to derive the lower bound of [12] is to compute the maximum distance a common node can move without changing its neighbor nodes which is regarded as a random variable $Z$ and calculate the expectation of $Z$. They treat the expectation of $Z$ as the lower bound for the location error of sensor nodes, which is computed as:

$$
E(Z)=\int_{0}^{\infty} z \dot{F}(z) d z=\frac{\pi r}{4 n_{d}}
$$

where $n_{d}$ is the node density.

From Eq.(19), we learn that the higher node density leads the higher localization accuracy. Although the virtual anchor nodes of our algorithm do not have the communication and sensing ability, their positions can be utilized as real anchor nodes to estimate those unknown-position nodes which are in the range of virtual anchor nodes. Thus, we conclude that the node density is increased for these unknown-position nodes after constructing the virtual anchor nodes and using the ME. Consequently, the location error of our proposed cooperative localization algorithm decreases with the increasing of node density under this situation.

\section{Communication Cost}

The number of messages exchanged a sensor node needs to transmit is treated as the communication cost in our localization algorithm. In our localization process, the anchor nodes will perform the broadcasting operation at every time slot. Thus the communication cost is related to the number of anchor nodes. In our proposed scheme, virtual anchor nodes cannot broadcast messages and will not increase communication cost. For those real anchor nodes, they will broadcast to unknown-position nodes a hello message with their ID, location and some recognize-bits which is totally only several bytes. For low anchor density, it is about hundred of bytes [15].

\section{NUMERICAL RESULTS}

In this section, simulation results are presented and analyzed. The performance evaluation focuses on the position estimation accuracy and localization coverage of the proposed algorithm with and without mobile element. In order to fair comparison, the total number of anchors for different algorithms is assigned identical. In the scenario of considering our proposed algorithm with $\mathrm{ME}$, we select one of the anchors as ME in this simulation work.
We consider an experiment region in a 2-dimensional region with a size of $100 \mathrm{~m} \times 100 \mathrm{~m}$. As can be seen from [3], centroid location is robust under the effect of the irregularity of the radio pattern. The reason is that the centroid algorithm does not depend on hop-count and HopSize such that the effect of irregularity is abated by the aggregation of beaconed information. Similar to [1],[4], we also assume the sensor nodes have the same maximum radio range $R$ at the beginning, which is used for normalization only. First, we deploy 300 sensor nodes randomly and $R$ is set to 25 meters. The number of sensor nodes and the radio range of sensor nodes will be increased then. The effect of degree of irregularity (DOI) on localization performance will be discussed later. All simulation results are averaged over 100 network scenarios.

Fig. 7 shows the localization error in percentage of the nominal radio range. As observed from Fig. 7, the proposed algorithm with $\mathrm{ME}$ has smaller localization error with higher anchor density. Generally, the localization error decreases as the number of anchor nodes increases. For the same number of anchor nodes and in a same WSN deployment, localization error incurred in the proposed scheme with one ME is smaller than that without ME. For example, with 25 anchor nodes, our proposed algorithm with one ME has an average error of approximately $0.45 R$, while the proposed algorithm without ME has an average error about $0.53 R$. Fig. 8 shows the localization coverage which is defined by Niculescu and Nath [4]. It is observed that our proposed algorithm with one ME achieves better localization coverage than the proposed localization algorithm without ME.

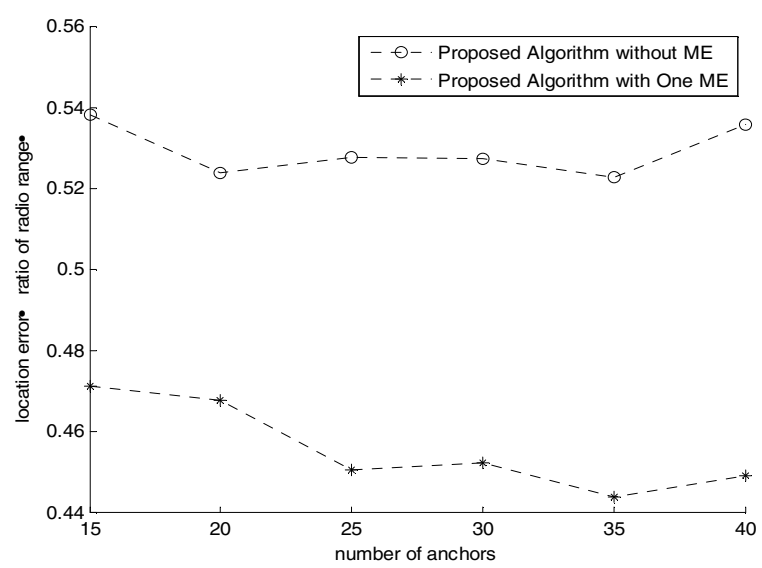

Fig.7. Localization error vs. number of anchors.

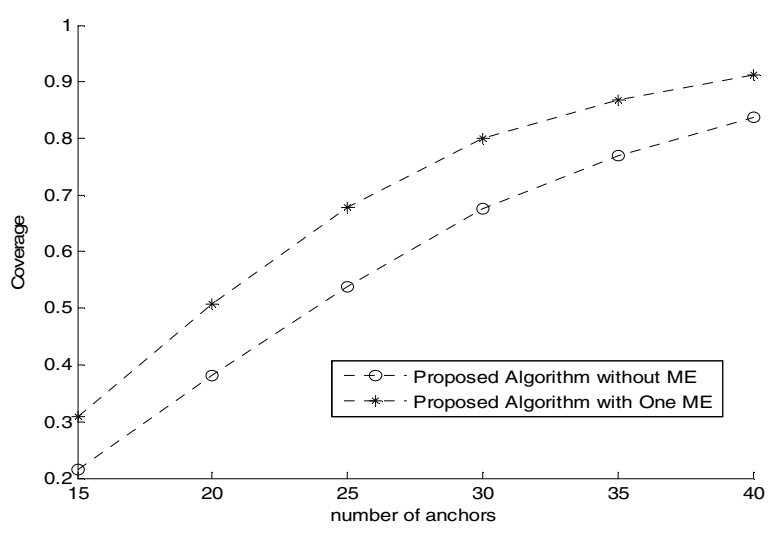

Fig.8. Localization coverage vs. number of anchors. 
The radio range of sensor nodes has an impact on the localization performance. Figs. 9 and 10 show the localization performance of both schemes in different node radio transmission range from 20 to 40 meters. As observed in Fig. 9 and Fig.10, the proposed algorithm with one ME achieves a better performance than the proposed localization algorithm without $\mathrm{ME}$ in general with respect to different radio range of sensor nodes in the systems. Herein, the number of anchor nodes and unknown-position nodes are fixed to 20 and 180 , respectively.

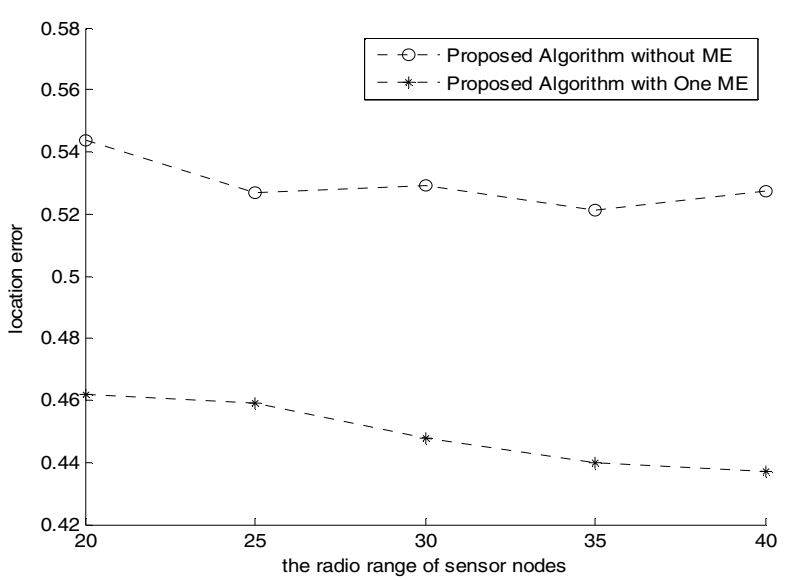

Fig.9. Localization error vs. radio range.

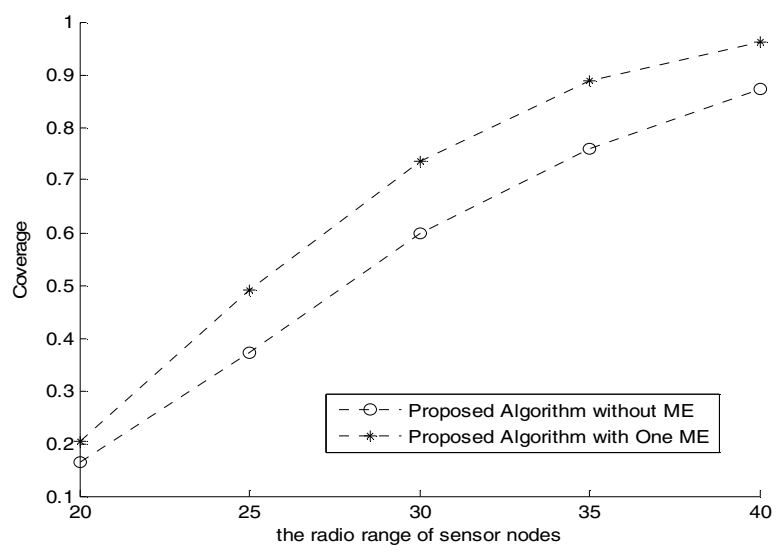

Fig.10. Localization coverage vs. radio range.

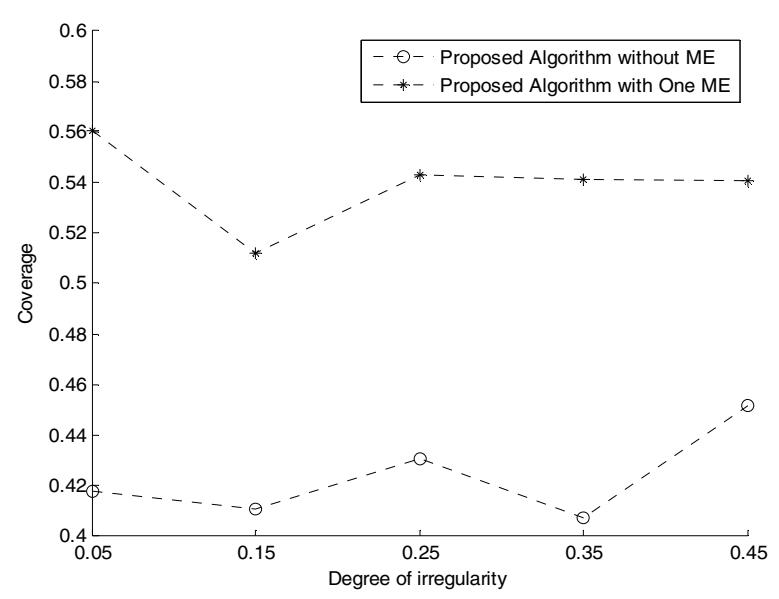

Fig.11. Localization coverage vs. DOI.

Variations in actual radio transmission patterns can have an impact on location performance. Unlike the perfect circles of radius $R$ assumed in our previous experiments, we use DOI to investigate the impact of irregular radio patterns on localization performance [3]. As can be seen from Fig.10, the localization performance of the localization algorithm varies as DOI increases. It is shown that the proposed algorithm with one ME still achieves a better localization performance than that without $\mathrm{ME}$ in general with respect to different DOIs. Herein, the number of unknown-position nodes is fixed to 270 and the number of anchor nodes is fixed to 30 .

\section{CONCLUSION}

We present a new cooperative localization scheme that can improve the location performance significantly in mobility-assisted wireless sensor networks. We develop an attractive movement strategy for MEs which can achieve an effective path under expected localization performance. Through using ME and virtual anchor nodes, the localization performance of the related location algorithm can be improved as shown in the simulation results. In future work, we intend to verify and improve the proposed cooperative localization scheme using real testbeds in mobility-assisted wireless sensor networks.

\section{REFERENCES}

[1] N. Bulusu, J. Heidemann, and D. Estrin, "GPS-less low cost outdoor localization for very small devices," IEEE Personal Communications Magazine, vol. 7, pp.28-34, October 2000.

[2] L. Girod and D. Estrin, "Robust range estimation using acoustic and multimodal sensing," in Proc. IEEE International Conference on Intelligent Robots and Systems, vol. 3, pp. 1312-1320, 2001.

[3] T. He, C. Huang, B.M. Blum, J. A. Stankovic, and T. Abdelzaher. "Range-free localization schemes for large scale sensor networks," in Proc. ACM MobiCom, San Diego, pp. 81-95, 2003.

[4] D. Niculescu and B. Nath. "Ad hoc positioning system (APS)," in Proc. IEEE GLOBECOM, San Antonio, pp. 2926-2931, 2001.

[5] X. Cheng, A. Thaeler, G. Xue, and D. Chen, "TPS: a time-based positioning scheme for outdoor wireless sensor networks," in Proc. IEEE INFOCOM, Hong Kong, China, pp. 2685-2696, March 2004.

[6] T.S. Rappapport, Wireless Communications: Principles and Practice, Prentice Hall: New Jersey, pp.50-143, 1996.

[7] G. Xing, J. Wang, K. Shen, Q. Huang, X. Jia and H.C. So, "Mobility-assisted spatiotemporal detection in wireless sensor networks," in Proc. 28th International Conference on Distributed Computing Systems (ICDCS), Beijing, China, Jun 17-20, 2008.

[8] P.X. Liu, X.M. Zhang, S. Tian, Z.W. Zhao, and P. Sun, "A novel virtual anchor node-based localization algorithm for wireless sensor networks", in Proc. of the Sixth International Conference on Networking, pp. 9-9,France,April 2007.

[9] J. Luo, H.V. Shukla, and J.-P. Hubaux, "Non-interactive location surveying for sensor neworks with mobility-differentiated ToA," in Proc. of the 25th IEEE INFOCOM, April 2006.

[10] M. Sichitiu and V. Ramadurai, "Localization of wireless sensor networks with a mobile beacon," in Proc. of the 1st IEEE MASS, 2004.

[11] P.N. Pathirana, N. Bulusu, A.V. Savkin, and S. Jha, "Node localization using mobile robots in delay-tolerant sensor networks," IEEE Trans. on Mobile Computing, vol. 4, no. 3, pp. 285-296, 2005.

[12] R. Nagpal, H. Shrobe, and J. Bachrach, "Organizing a global coordinate system from local information on an ad hoc sensor network," in Proc. Second International Workshop on Information Processing in Sensor Networks (IPSN), pp.333 - 348, 2003.

[13] M. Rudafshani and S. Datta, "Localization in wireless sensor networks," in Proceedings of the 6th International Conference on Information Processing in Sensor Networks (IPSN'07), pp.51-60, Cambridge, Massachusetts, USA, 2007.

[14] W. Mendenhall, D. Wackerly, and R. Scheaffer. Mathematical Statistics with Applications, PWS-Kent Publishing Company, 1989.

[15] J. Luo and Q. Zhang, "Relative distance based localization for mobile sensor networks," in Proc. IEEE GLOBECOM, pp.1076-1080, Nov. 2007.

[16] B. Xiao, H. Chen, and S. Zhou, "Distributed localization using a moving beacon in wireless sensor networks," IEEE Transactions on Parallel and Distributed Systems, vol. 19, no. 5, pp.587-600, May, 2008. 